\title{
Extension Operators Preserving Janowski Classes of Univalent Functions
}

\author{
Andra Manu
}

\begin{abstract}
In this paper, our main interest is devoted to study the extension operator $\Phi_{n, \alpha, \beta}: \mathcal{L} S \rightarrow \mathcal{L} S_{n}$ given by $\Phi_{n, \alpha, \beta}(f)(z)=\left(f\left(z_{1}\right), \widetilde{z}\left(f\left(z_{1}\right) / z_{1}\right)^{\alpha}\left(f^{\prime}\left(z_{1}\right)\right)^{\beta}\right)$, $z=\left(z_{1}, \widetilde{z}\right) \in \mathbf{B}^{n}$, where $\alpha, \beta \geq 0$. We shall prove that if $f \in S$ can be embedded as the first element of a $g$-Loewner chain with $g: U \rightarrow \mathbb{C}$ given by $g(\zeta)=(1+A \zeta) /(1+B \zeta)$, $|\zeta|<1$, and $-1 \leq B<A \leq 1$, then $F=\Phi_{n, \alpha, \beta}(f)$ can be embedded as the first element of a $g$-Loewner chain on the unit ball $\mathbf{B}^{n}$ for $\alpha \in[0,1], \beta \in[0,1 / 2]$ and $\alpha+\beta \leq 1$. As a consequence, the operator $\Phi_{n, \alpha, \beta}$ preserves the notions of Janowski starlikeness on $\mathbf{B}^{n}$ and Janowski almost starlikeness on $\mathbf{B}^{n}$. Particular cases will be also mentioned.

On the other hand, we are also concerned about some radius problems related to the operator $\Phi_{n, \alpha, \beta}$ and the Janowski class $S^{*}(a, b)$. We compute the radius $S^{*}(a, b)$ of the class $S$ (respectively $S^{*}$ ).
\end{abstract}

\section{Introduction and preliminaries}

Let $\mathbb{C}^{n}$ denote the space of $n$ complex variables $z=\left(z_{1}, z_{2}, \ldots, z_{n}\right)$ where $z_{j} \in \mathbb{C}, 1 \leq$ $j \leq n$ with the Euclidean inner product $\langle z, w\rangle=\sum_{j=1}^{n} z_{j} \bar{w}_{j}$ and the Euclidean norm $\|z\|=\sqrt{\langle z, z\rangle}$. The open unit ball $\left\{z \in \mathbb{C}^{n}:\|z\|=1\right\}$ is denoted by $\mathbf{B}^{n}$ and, in the case of one complex variable, $\mathbf{B}^{1}$ is denoted by $U$. We denote by $H\left(\mathbf{B}^{n}\right)$ the set of holomorphic mappings from $\mathbf{B}^{n}$ into $\mathbb{C}^{n}$. We say that $f \in H\left(\mathbf{B}^{n}\right)$ is normalized if $f(0)=0$ and $D f(0)=I_{n}$, where $I_{n}$ is the $n \times n$-unitary matrix. We denote by $\mathcal{L} S_{n}$ the set of normalized locally biholomorphic mappings on $\mathbf{B}^{n}$ and, in the case of one complex variable, $\mathcal{L} S_{1}$ is denoted by $\mathcal{L} S$. We consider the following notations: $S\left(\mathbf{B}^{n}\right)$ the family of normalized biholomorphic mappings on $\mathbf{B}^{n}, S^{*}\left(\mathbf{B}^{n}\right)$ the family of normalized biholomorphic mappings that are starlike with respect to zero, respectively $K\left(\mathbf{B}^{n}\right)$ the family of normalized biholomorphic mappings on $\mathbf{B}^{n}$ that are convex. In the case of one complex variable, the above families will be denoted by $S, S^{*}$, respectively $K$.

Further we will introduce some subclasses of $H\left(\mathbf{B}^{n}\right)$ that will be useful in the next sections.

Received May 15, 2018; Accepted April 14, 2019.

Communicated by Xiang Fang.

2010 Mathematics Subject Classification. Primary: 32H99; Secondary: 30C45.

Key words and phrases. $g$-Loewner chain, $g$-parametric representation, $g$-starlikeness, $g$-spirallikeness, Janowski starlikeness, Janowski almost starlikeness, Roper-Suffridge extension operator. 
The Carathéodory class of holomorphic functions with positive real part on $U$ is defined by (see e.g., [24])

$$
\mathcal{P}=\{p \in H(U): p(0)=1, \operatorname{Re} p(z)>0,|z|<1\}
$$

The above class was generalized to the unit ball $\mathbf{B}^{n}(n \geq 2)$ as follows (see 23):

$$
\mathcal{M}=\left\{h \in H\left(\mathbf{B}^{n}\right): h(0)=0, D h(0)=I_{n}, \operatorname{Re}\langle h(z), z\rangle>0, z \in \mathbf{B}^{n} \backslash\{0\}\right\} .
$$

This class is related to some subclasses of biholomorphic mappings on $\mathbf{B}^{n}$, for example the class of biholomorphic mappings which have parametric representation, the class of starlike mappings and the class of spirallike mappings of type $\delta$, where $\delta \in(-\pi / 2, \pi / 2)$ (see e.g., 10,28 and the references therein).

Definition 1.1. Let $g: U \rightarrow \mathbb{C}$ be an univalent function on the unit disk $U$ such that $g(0)=1, \operatorname{Re} g(\zeta)>0, \zeta \in U$ and the coefficients in its power series expansion are real (i.e., $g(\bar{\zeta})=\overline{g(\zeta)}$ on $U$ ). Also, assume $g$ satisfies the following conditions for all $r \in(0,1)$ :

$$
\begin{aligned}
& \min _{|\zeta|=r} \operatorname{Re} g(\zeta)=\min \{g(r), g(-r)\} \\
& \max _{|\zeta|=r} \operatorname{Re} g(\zeta)=\max \{g(r), g(-r)\}
\end{aligned}
$$

In this paper, our main concern is the case when the function $g$ has the following particular form:

$$
g(\zeta)=\frac{1+A \zeta}{1+B \zeta}, \quad|\zeta|<1, \quad \text { where }-1 \leq B<A \leq 1
$$

Let $\mathcal{M}_{g}$ be the subclass of $\mathcal{M}$ given by (see 7$]$ )

$$
\mathcal{M}_{g}=\left\{h \in H\left(\mathbf{B}^{n}\right): h(0)=0, D h(0)=I_{n},\left\langle h(z), z /\|z\|^{2}\right\rangle \in g(U), z \in \mathbf{B}^{n} \backslash\{0\}\right\},
$$

where $g$ is given as in Definition 1.1. Note that if $h \in \mathcal{M}_{g}$, then $\left.\left\langle h(z), z /\|z\|^{2}\right\rangle\right|_{z=0}=1$, since $h(0)=0$ and $D h(0)=I_{n}$.

Let $g: U \rightarrow \mathbb{C}$ be a function given by (1.1) then we obtain the following particular forms of $\mathcal{M}_{g}$ by choosing suitable values of parameters $A$ and $B$.

Case $I: B=-1$. In this situation, we have that

$$
\mathcal{M}_{g}=\left\{h \in H\left(\mathbf{B}^{n}\right): h(0)=0, D h(0)=I_{n}, \operatorname{Re}\langle h(z), z\rangle>\frac{1-A}{2}\|z\|^{2}, z \in \mathbf{B}^{n} \backslash\{0\}\right\} .
$$

Moreover, if $A=1$, then $\mathcal{M}_{g}=\mathcal{M}$.

Case II: $B \neq-1$. In this case, we have that

$$
\mathcal{M}_{g}=\left\{h \in H\left(\mathbf{B}^{n}\right): h(0)=0, D h(0)=I_{n},\left|\frac{1}{\|z\|^{2}}\langle h(z), z\rangle-\frac{1-A B}{1-B^{2}}\right|<\frac{A-B}{1-B^{2}}, z \in \mathbf{B}^{n} \backslash\{0\}\right\} .
$$


Now, we assume that $A=(a-1) / b$ and $B=\left(a^{2}-b^{2}-a\right) / b$, where $|1-a|<b \leq a$. In the case that $b<a$, we obtain that

$$
\mathcal{M}_{g}=\left\{h \in H\left(\mathbf{B}^{n}\right): h(0)=0, D h(0)=I_{n},\left|\frac{1}{\|z\|^{2}}\langle h(z), z\rangle-\frac{a}{a^{2}-b^{2}}\right|<\frac{b}{a^{2}-b^{2}}, z \in \mathbf{B}^{n} \backslash\{0\}\right\} .
$$

This special case is related to Janowski starlikeness on $\mathbf{B}^{n}$ (see [4]).

If $A=\left(a-a^{2}+b^{2}\right) / b$ and $B=(1-a) / b$ with $|1-a|<b \leq a$, then

$$
\mathcal{M}_{g}=\left\{h \in H\left(\mathbf{B}^{n}\right): h(0)=0, D h(0)=I_{n},\left|\frac{1}{\|z\|^{2}}\langle h(z), z\rangle-a\right|<b, z \in \mathbf{B}^{n} \backslash\{0\}\right\} .
$$

This case is related to Janowski almost starlikeness on $\mathbf{B}^{n}$ (see [4]).

The following subclasses of biholomorphic mappings on $\mathbf{B}^{n}$ were introduced by Curt (see [4]).

Definition 1.2. (see [4]) Assume $a, b \in \mathbb{R}$ such that $|1-a|<b \leq a$. Let

$$
S^{*}\left(a, b, \mathbf{B}^{n}\right)=\left\{f \in \mathcal{L} S_{n}:\left|\frac{\|z\|^{2}}{\left\langle[D f(z)]^{-1} f(z), z\right\rangle}-a\right|<b, z \in \mathbf{B}^{n} \backslash\{0\}\right\}
$$

be the class of Janowski starlike mappings on $\mathbf{B}^{n}$ and let

$$
\mathcal{A} S^{*}\left(a, b, \mathbf{B}^{n}\right)=\left\{f \in \mathcal{L} S_{n}:\left|\frac{\left\langle[D f(z)]^{-1} f(z), z\right\rangle}{\|z\|^{2}}-a\right|<b, z \in \mathbf{B}^{n} \backslash\{0\}\right\}
$$

be the class of Janowski almost starlike mappings on $\mathbf{B}^{n}$.

We remark that both classes $S^{*}\left(a, b, \mathbf{B}^{n}\right)$ and $\mathcal{A} S^{*}\left(a, b, \mathbf{B}^{n}\right)$ are subsets of $S^{*}\left(\mathbf{B}^{n}\right)$, since $|a-1|<b \leq a$.

The class $S^{*}\left(a, b, \mathbf{B}^{1}\right)$ reduces to the following subclass of $S^{*}$ :

$$
S^{*}(a, b)=\left\{f \in H(U): f(0)=0, f^{\prime}(0)=1,\left|\frac{z f^{\prime}(z)}{f(z)}-a\right|<b, z \in U\right\} .
$$

Note that the class $S^{*}(a, b)$ was introduced by Silverman in 26] (see also 27]). This class is closely related to the following class of holomorphic functions on $U$, which was introduced by Janowski 16

$$
S^{*}[A, B]=\left\{f \in H(U): f(0)=0, f^{\prime}(0)=1, \frac{z f^{\prime}(z)}{f(z)} \prec \frac{1+A z}{1+B z}, z \in U\right\},
$$

where $-1 \leq B<A \leq 1$ and " $\prec$ " is the usual symbol of subordination.

Also, the class $\mathcal{A} S^{*}\left(a, b, \mathbf{B}^{1}\right)$ reduces to the following subclass of $S^{*}$ :

$$
\mathcal{A} S^{*}(a, b)=\left\{f \in H(U): f(0)=0, f^{\prime}(0)=1,\left|\frac{f(z)}{z f^{\prime}(z)}-a\right|<b, z \in U\right\} .
$$

Next, we recall the definition of starlikeness of order $\gamma$ on $\mathbf{B}^{n}$, where $\gamma \in[0,1)$. This notion was introduced by Curt [3] and Kohr [17]. 
Definition 1.3. Let $f \in \mathcal{L} S_{n}$ and $\gamma \in[0,1)$. The mapping $f$ is said to be starlike of order $\gamma$ if

$$
\operatorname{Re}\left\{\frac{\|z\|^{2}}{\left\langle[D f(z)]^{-1} f(z), z\right\rangle}\right\}>\gamma, \quad z \in \mathbf{B}^{n} \backslash\{0\} .
$$

Let $S_{\gamma}^{*}\left(\mathbf{B}^{n}\right)$ be the set of starlike mappings of order $\gamma$ on $\mathbf{B}^{n}$.

Now, we recall the notion of almost starlikeness of order $\gamma$ on $\mathbf{B}^{n}$, where $\gamma \in[0,1)$. This notion was introduced by Kohr [18] for $\gamma=1 / 2$, Feng [6], and by Xu and Liu [31].

Definition 1.4. Let $f \in \mathcal{L} S_{n}$ and $\gamma \in[0,1)$. The mapping $f$ is said to be almost starlike of order $\gamma$ on $\mathbf{B}^{n}$ if

$$
\operatorname{Re}\left\{\frac{\left\langle[D f(z)]^{-1} f(z), z\right\rangle}{\|z\|^{2}}\right\}>\gamma, \quad z \in \mathbf{B}^{n} \backslash\{0\} .
$$

Let $\mathcal{A} S_{\gamma}^{*}\left(\mathbf{B}^{n}\right)$ be the set of almost starlike mappings of order $\gamma$ on $\mathbf{B}^{n}$.

Remark 1.5. It is easy to see that if $a=b=1 /(2 \gamma)$, where $\gamma \in(0,1)$, then

$$
\mathcal{A} S^{*}\left(\frac{1}{2 \gamma}, \frac{1}{2 \gamma}, \mathbf{B}^{n}\right)=S_{\gamma}^{*}\left(\mathbf{B}^{n}\right) \quad \text { and } \quad S^{*}\left(\frac{1}{2 \gamma}, \frac{1}{2 \gamma}, \mathbf{B}^{n}\right)=\mathcal{A} S_{\gamma}^{*}\left(\mathbf{B}^{n}\right) \text {. }
$$

Remark 1.6. Let $f \in \mathcal{L} S_{n}$ and $h(z)=[D f(z)]^{-1} f(z), z \in \mathbf{B}^{n}$. Also, let $a, b \in \mathbb{R}$ be such that $|a-1|<b \leq a$ and $\gamma \in[0,1)$. In view of [4, Remark 3.3], we deduce the following relations:

(i) $f \in S^{*}\left(a, b, \mathbf{B}^{n}\right) \Longleftrightarrow h \in \mathcal{M}_{g}$, where $g(\zeta)=\frac{1+(a-1) / b \zeta}{1+\left(a^{2}-b^{2}-a\right) / b \zeta},|\zeta|<1$.

(ii) $f \in \mathcal{A} S^{*}\left(a, b, \mathbf{B}^{n}\right) \Longleftrightarrow h \in \mathcal{M}_{g}$, where $g(\zeta)=\frac{1+\left(a-a^{2}+b^{2}\right) / b \zeta}{1+(1-a) / b \zeta},|\zeta|<1$.

(iii) $f \in S_{\gamma}^{*}\left(\mathbf{B}^{n}\right) \Longleftrightarrow h \in \mathcal{M}_{g}$, where $g(\zeta)=\frac{1+\zeta}{1+(2 \gamma-1) \zeta},|\zeta|<1$.

(iv) $f \in \mathcal{A} S_{\gamma}^{*}\left(\mathbf{B}^{n}\right) \Longleftrightarrow h \in \mathcal{M}_{g}$, where $g(\zeta)=\frac{1+(1-2 \gamma) \zeta}{1-\zeta},|\zeta|<1$.

Further, we present the notion of $g$-starlikeness on $\mathbf{B}^{n}$, introduced by Graham, Hamada and Kohr in [7] (see also [13).

Definition 1.7. Let $g: U \rightarrow \mathbb{C}$ be a function given by Definition 1.1. A mapping $f \in \mathcal{L} S_{n}$ is said to be $g$-starlike on $\mathbf{B}^{n}$ if $h \in \mathcal{M}_{g}$ where $h(z)=[D f(z)]^{-1} f(z)$ for all $z \in \mathbf{B}^{n}$. We denote by $S_{g}^{*}\left(\mathbf{B}^{n}\right)$ the class of $g$-starlike mappings on $\mathbf{B}^{n}$ and $S_{g}^{*}\left(\mathbf{B}^{1}\right)$ by $S_{g}^{*}$.

Taking into account the analytical characterization of starlikeness on $\mathbf{B}^{n}$ due to Suffridge [28], it is easy to see that $S_{g}^{*}\left(\mathbf{B}^{n}\right)$ is a subset of $S^{*}\left(\mathbf{B}^{n}\right)$.

Next, we will present some observations regarding the case of $g$-starlikeness on the complex plane. The purpose of this remark is to point out how the class $S_{g}^{*}$ can be related to the Janowski class $S^{*}[A, B]$, respectively to the class $S^{*}(a, b)$ introduced by Silverman, in the case that the function $g$ is given by the relation (1.1). 
Remark 1.8. Let $A, B \in \mathbb{R}$ be such that $-1 \leq B<A \leq 1$. Also, let $g(\zeta)=(1+A \zeta) /(1+$ $B \zeta), \zeta \in U$. The class $S_{g}^{*}$ can be rewritten as follows:

(i) $S_{g}^{*}=\left\{f \in H(U): f(0)=0, f^{\prime}(0)=1, f(z) /\left(z f^{\prime}(z)\right) \prec(1+A z) /(1+B z), z \in U\right\}$.

(ii) $S_{g}^{*}=S^{*}[-B,-A]$.

(iii) If $A \neq 1$ then $S_{g}^{*}=S^{*}(a, b)$, where $a=(1-A B) /\left(1-A^{2}\right), b=(A-B) /\left(1-A^{2}\right)$. If $A=1$ then $S_{g}^{*}=S_{(1+B) / 2}^{*}$.

Proof. Indeed, we know from the definition of $S_{g}^{*}$ that

$$
S_{g}^{*}=\left\{f \in H(U): f(0)=0, f^{\prime}(0)=1, \frac{f(z)}{z f^{\prime}(z)} \in g(U),|z|<1\right\} .
$$

The condition $f(z) /\left(z f^{\prime}(z)\right) \in g(U)$ is equivalent to $f(z) /\left(z f^{\prime}(z)\right) \prec(1+A z) /(1+B z)$, so this justifies (i).

On the other hand, if $f \in H(U), f(0)=0$ and $f^{\prime}(0)=1$ then $f \in S_{g}^{*}$ if and only if $z f^{\prime}(z) / f(z) \prec(1-B z) /(1-A z)$. Also, it is easy to see that $-1 \leq-A<-B \leq 1$. This proves (ii). If $A \neq 1$ then $q$ maps the unit disk $U$ onto the open disk $U((1-A B) /(1-$ $\left.\left.A^{2}\right),(A-B) /\left(1-A^{2}\right)\right)$. If $A=1$, the unit disk $U$ is mapped onto $\{\zeta \in \mathbb{C}: \operatorname{Re} \zeta>$ $(1+B) / 2\}$. Thus, the statement of Remark 1.8 (iii) is now justified.

Next, we take into consideration the case $n \geq 2$. In [4, Remark 3.3], Curt obtained the appropriate values of parameters $A$ and $B$ such that the classes $S^{*}\left(a, b, \mathbf{B}^{n}\right)$ and $\mathcal{A} S^{*}\left(a, b, \mathbf{B}^{n}\right)$ can be rewritten as $S_{g}^{*}\left(\mathbf{B}^{n}\right)$.

Remark 1.9. (see [4]) Let $a, b \in \mathbb{R}$ be such that $|1-a|<b \leq a$ and let the function $g$ be given by $g(\zeta)=(1+A \zeta) /(1+B \zeta), \zeta \in U$, where $-1 \leq B<A \leq 1$.

(i) If $A=(a-1) / b, B=\left(a^{2}-b^{2}-a\right) / b$ then $S^{*}\left(a, b, \mathbf{B}^{n}\right)=S_{g}^{*}\left(\mathbf{B}^{n}\right)$.

(ii) If $A=\left(a-a^{2}+b^{2}\right) / b, B=(1-a) / b$ then $\mathcal{A} S^{*}\left(a, b, \mathbf{B}^{n}\right)=S_{g}^{*}\left(\mathbf{B}^{n}\right)$.

Chirilă [2] introduced the notion of $g$-spirallikeness of type $\delta$, where $\delta \in(-\pi / 2, \pi / 2)$. The particular case when $g(\zeta)=(1+\zeta) /(1-\zeta), \zeta \in U$ was studied in [14].

Definition 1.10. Let $f \in \mathcal{L} S_{n}$ and let $g: U \rightarrow \mathbb{C}$ be a function given by Definition 1.1 . We say that $f$ is $g$-spirallike of type $\delta$, where $\delta \in(-\pi / 2, \pi / 2)$, if $h(\cdot, t) \in \mathcal{M}_{g}, t \geq 0$, where

$$
h(z, t)=i a z+(1-i a) e^{-i a t}\left[D f\left(e^{i a t} z\right)\right]^{-1} f\left(e^{i a t} z\right), \quad z \in \mathbf{B}^{n}, t \geq 0,
$$

where $a=\tan \delta$. 
Remark 1.11. Let $g: U \rightarrow \mathbb{C}$ be the function given by $g(\zeta)=(1+A \zeta) /(1+B \zeta), \zeta \in U$. The class of $g$-spirallike mappings of type $\delta$ reduces to some well known classes of biholomorphic mappings on $\mathbf{B}^{n}$ by choosing suitable values for the parameters $A$ and $B$. In particular, if $A=1$ and $B=-1$ then the class of $g$-spirallike mappings of type $\delta$ reduces to the class of spirallike mappings of type $\delta$ on $\mathbf{B}^{n}$, which is denoted by $\widehat{S}_{\delta}\left(\mathbf{B}^{n}\right)$. This class was introduced in [14]. For $A=1$ and $B=2 \gamma-1$ with $\gamma \in(0,1)$, we obtain the class of spirallike mappings of type $\delta$ and order $\gamma$ on $\mathbf{B}^{n}$ (see $[20]$ ). The case of $\delta=0$ in Definition 1.10 leads us to the class of $g$-starlike mappings on $\mathbf{B}^{n}$.

We need to recall the definition of a Loewner chain prior introducing the notion of $g$-parametric representation. Many results related to Loewner chains in $\mathbb{C}^{n}$ may be found in $5,7,10,12,23,29$.

Definition 1.12. (see [23]) Let $f, g \in H\left(\mathbf{B}^{n}\right)$. We say that $f$ is subordinate to $g$ (write $f \prec g)$ if there is a Schwarz mapping $v$ (i.e., $\left.v \in H\left(\mathbf{B}^{n}\right),\|v(z)\| \leq\|z\|, z \in \mathbf{B}^{n}\right)$ such that $f(z)=g(v(z)), z \in \mathbf{B}^{n}$.

Definition 1.13. (see [23]) Let $f: \mathbf{B}^{n} \times[0, \infty) \rightarrow \mathbb{C}^{n}$. We say that $f$ is a Loewner chain if $f(\cdot, t)$ is biholomorphic on $\mathbf{B}^{n}, f(0, t)=0, D f(0, t)=e^{t} I_{n}$ for $t \geq 0$ and $f(\cdot, s) \prec f(\cdot, t)$ whenever $0 \leq s \leq t<\infty$.

Further, we will present a characterization of Loewner chain obtained by Pfaltzgraff 23 .

Lemma 1.14. Let $f=f(z, t): \mathbf{B}^{n} \times[0, \infty) \rightarrow \mathbb{C}^{n}$ be a mapping such that $f(\cdot, t) \in H\left(\mathbf{B}^{n}\right)$, $f(0, t)=0, D f(0, t)=e^{t} I_{n}$ for $t \geq 0$ and $f(z, \cdot)$ is locally absolutely continuous on $[0, \infty)$ locally uniformly with respect to $z \in \mathbf{B}^{n}$. Assume that there exists a mapping $h=h(z, t): \mathbf{B}^{n} \times[0, \infty) \rightarrow \mathbb{C}^{n}$ which satisfies the following conditions:

(i) $h(\cdot, t) \in \mathcal{M}$ for $t \geq 0$,

(ii) $h(z, \cdot)$ is measurable on $[0, \infty)$ for $z \in \mathbf{B}^{n}$,

and such that the following differential equation is fulfilled

$$
\frac{\partial f}{\partial t}(z, t)=D f(z, t) h(z, t) \quad \text { a.e. } t \geq 0, \forall z \in \mathbf{B}^{n} .
$$

Further, assume that $\left\{e^{-t} f(\cdot, t)\right\}_{t \geq 0}$ is a locally uniformly bounded family on $\mathbf{B}^{n}$. Then $f(z, t)$ is a Loewner chain.

Definition 1.15. (see [5]) A mapping $h(z, t): \mathbf{B}^{n} \times[0, \infty) \rightarrow \mathbb{C}^{n}$ which satisfies the conditions (i) and (ii) in Lemma 1.14 is called a Herglotz vector field. 
The notions of $g$-Loewner chain and $g$-parametric representation were introduced by Graham, Hamada and Kohr in [7, where the function $g$ satisfies the conditions of Definition 1.1.

Definition 1.16. Given a mapping $f=f(z, t): \mathbf{B}^{n} \times[0, \infty) \rightarrow \mathbb{C}^{n}$, one says that $f$ is a $g$-Loewner chain if $f(z, t)$ is a Loewner chain such that $\left\{e^{-t} f(\cdot, t)\right\}_{t \geq 0}$ is a normal family on unit ball $\mathbf{B}^{n}$ and the mapping $h=h(z, t)$, which occurs in the following Loewner differential equation

$$
\frac{\partial f}{\partial t}(z, t)=D f(z, t) h(z, t) \quad \text { a.e. } t \geq 0, \forall z \in \mathbf{B}^{n}
$$

satisfies the condition $h(\cdot, t) \in \mathcal{M}_{g}$ for a.e. $t \geq 0$.

Definition 1.17. Given a normalized holomorphic mapping $f: \mathbf{B}^{n} \rightarrow \mathbb{C}^{n}$, we say that $f$ has $g$-parametric representation if there exists a $g$-Loewner chain $f(z, t)$ such that $f$ can be embedded as the first element of the $g$-Loewner chain $f(z, t)$ (i.e., $f=f(\cdot, 0)$ ). We will denote by $S_{g}^{0}\left(\mathbf{B}^{n}\right)$ the set of mappings which have $g$-parametric representation on $\mathbf{B}^{n}$.

We remark that if $g(\zeta)=(1+\zeta) /(1-\zeta), \zeta \in U$, then $S_{g}^{0}\left(\mathbf{B}^{n}\right)$ reduces to the set $S^{0}\left(\mathbf{B}^{n}\right)$ of mappings which have parametric representation, hence any Loewner chain $f(z, t)$ on $\mathbf{B}^{n}$, such that $\left\{e^{-t} f(\cdot, t)\right\}_{t \geq 0}$ is a normal family on $\mathbf{B}^{n}$, is a $g$-Loewner chain on $\mathbf{B}^{n}$ (see $[7]$. Also, the family $S_{g}^{0}\left(\mathbf{B}^{n}\right)$ where the function $g$ is given by $g(\zeta)=\frac{1+\zeta}{1+(2 \gamma-1) \zeta}$, $\zeta \in U$ and $\gamma \in(0,1)$ was studied by Chirilă in [1].

Remark 1.18. (i) Let $f \in \mathcal{L} S_{n}$. We have that $f \in S_{g}^{*}\left(\mathbf{B}^{n}\right)$ if and only if $f(z, t)=e^{t} f(z)$ is a $g$-Loewner chain for all $z \in \mathbf{B}^{n}$ and $t \geq 0$ (see [7]).

(ii) Chirilă in [2, Teorem 3.1] proved that if $f \in \mathcal{L} S_{n}$ and $\delta \in(-\pi / 2, \pi / 2)$, then $f$ is $g$-spirallike of type $\delta$ if and only if $f(z, t)=e^{(1-i a) t} f\left(e^{i a t} z\right)$ is a $g$-Loewner chain, where $a=\tan \delta$.

Let $\Phi_{n, \alpha, \beta}$ be the extension operator defined by the following relation (see [8]):

$$
\Phi_{n, \alpha, \beta}(f)(z)=\left(f\left(z_{1}\right), \widetilde{z}\left(\frac{f\left(z_{1}\right)}{z_{1}}\right)^{\alpha}\left(f^{\prime}\left(z_{1}\right)\right)^{\beta}\right), \quad z=\left(z_{1}, \widetilde{z}\right) \in \mathbf{B}^{n}
$$

where $\alpha \geq 0, \beta \geq 0$ and $f \in \mathcal{L} S$ such that $f\left(z_{1}\right) \neq 0, z \in U \backslash\{0\}$.

The branches of the power functions are chosen such that

$$
\left.\left(\frac{f\left(z_{1}\right)}{z_{1}}\right)^{\alpha}\right|_{z_{1}=0}=1,\left.\quad\left(f^{\prime}\left(z_{1}\right)\right)^{\beta}\right|_{z_{1}=0}=1 .
$$

We observe that for $\alpha=0, \beta=1 / 2$, the operator $\Phi_{n, \alpha, \beta}$ reduces to the Roper-Suffridge operator $\Phi_{n}: \mathcal{L} S \rightarrow \mathcal{L} S_{n}$, given by (see 25$]$ )

$$
\Phi_{n}(f)(z)=\left(f\left(z_{1}\right), \widetilde{z} \sqrt{f^{\prime}\left(z_{1}\right)}\right), \quad z=\left(z_{1}, \widetilde{z}\right) \in \mathbf{B}^{n}
$$


It is known that the operator $\Phi_{n, \alpha, \beta}$ preserves the notions of starlikeness and parametric representation from unit disk $U$ into the unit ball $\mathbf{B}^{n}$, for $n \geq 2$ and $\alpha \in[0,1], \beta \in[0,1 / 2]$, $\alpha+\beta \leq 1$ (see 8 ). But $\Phi_{n, \alpha, \beta}$ preserves the notion of convexity from unit disk $U$ into the unit ball $\mathbf{B}^{n}$ if and only if $(\alpha, \beta)=(0,1 / 2)$ (see 8,25$]$ ). Various properties of the operator $\Phi_{n, \alpha, \beta}$ were investigated in [11,21], in the case $\alpha=0$ and $\beta \in[0,1 / 2]$ (see also [9], in the case $\beta=0)$. Also, the operator $\Phi_{n, \alpha, \beta}$ was studied in [1,8,10].

In this paper, we continue the work in $[1,2,7,8$ concerning extension operators and $g$-Loewner chains in $\mathbb{C}^{n}$. We consider the operator $\Phi_{n, \alpha, \beta}$ and the set $S_{g}^{0}(U)$ of normalized holomorphic functions on unit disk $U$ that have $g$-parametric representation, where $g(\zeta)=$ $(1+A \zeta) /(1+B \zeta),|\zeta|<1$ and $-1 \leq B<A \leq 1$.

We shall prove that if $f \in S$ can be embedded as first element of a $g$-Loewner chain, where $g: U \rightarrow \mathbb{C}$ is given by the relation (1.1), then $F=\Phi_{n, \alpha, \beta}(f)$ can be embedded as first element of a $g$-Loewner chain on the unit ball $\mathbf{B}^{n}$ for $\alpha \in[0,1], \beta \in[0,1 / 2]$ and $\alpha+\beta \leq 1$. As a consequence, the operator $\Phi_{n, \alpha, \beta}$ preserves the notions of Janowski starlikeness and Janowski almost starlikeness from the unit disk into the unit ball $\mathbf{B}^{n}$. Particular cases from [1,2] will be also mentioned.

In the last part of the paper, we obtain some radius of Janowski starlikeness associated to some classes of biholomorphic mappings on $\mathbf{B}^{n}$ generated by the extension operator $\Phi_{n, \alpha, \beta}$.

\section{Main results}

In this section we prove the following theorem, which is the main result of this paper. The following result was obtained in [8, Theorem 2.1] (see also 11 for $\alpha=0$ ), in the case that $g(\zeta)=(1+\zeta) /(1-\zeta), \zeta \in U$. Also, Theorem 2.1 was recently obtained by Chirilă (see [1]) when the function $g$ is given by $g(\zeta)=\frac{1+\zeta}{1+(2 \gamma-1) \zeta}, \zeta \in U$, where $\gamma \in(0,1)$.

Theorem 2.1. Let $g: U \rightarrow \mathbb{C}$ be the function given by $g(\zeta)=(1+A \zeta) /(1+B \zeta), \zeta \in U$, where $-1 \leq B<A \leq 1$. If $f \in S$ has g-parametric representation, then $F=\Phi_{n, \alpha, \beta}(f)$ also has g-parametric representation on unit ball $\mathbf{B}^{n}$ for $\alpha \in[0,1], \beta \in[0,1 / 2]$ and $\alpha+\beta \leq 1$.

Proof. In order to prove the result, we shall use arguments similar to those used in 8 , Theorem 2.1] and [1, Theorem 2.1]. It is obvious that it is enough to consider only the case $n=2$.

We know that $f$ can be embedded as first element of a $g$-Loewner chain, therefore there exists a $g$-Loewner chain $f\left(z_{1}, t\right)$ such that $f\left(z_{1}, 0\right)=f\left(z_{1}\right), z_{1} \in U$.

Let us consider the following mapping $F_{\alpha, \beta}: \mathbf{B}^{2} \times[0, \infty) \rightarrow \mathbb{C}^{2}$, defined by

$$
F_{\alpha, \beta}(z, t)=\left(f\left(z_{1}, t\right), e^{(1-\alpha-\beta) t} z_{2}\left(\frac{f\left(z_{1}, t\right)}{z_{1}}\right)^{\alpha}\left(f^{\prime}\left(z_{1}, t\right)\right)^{\beta}\right)
$$


for $z=\left(z_{1}, z_{2}\right) \in \mathbf{B}^{2}, t \geq 0$. As it follows from [8], $F_{\alpha, \beta}(z, t)$ is a Loewner chain, since $\alpha \in[0,1], \beta \in[0,1 / 2]$ and $\alpha+\beta \leq 1$.

We know that given a Loewner chain $f\left(z_{1}, t\right)$ on $U$, there exists a function $p(\cdot, t)$ that belongs to $H(U)$ for $t \geq 0$, is measurable in $t \geq 0$, with $p(0, t)=1, \operatorname{Re} p\left(z_{1}, t\right)>0, z_{1} \in U$, $0 \leq t<\infty$, and such that

$$
\frac{\partial f}{\partial t}\left(z_{1}, t\right)=z_{1} f^{\prime}\left(z_{1}, t\right) p\left(z_{1}, t\right) \quad \text { a.e. } t \geq 0, \forall z_{1} \in U
$$

Also, the fact that $f\left(z_{1}, t\right)$ is a $g$-Loewner chain implies that $p\left(z_{1}, t\right) \in g(U)$ for a.e. $t \geq 0$, $\forall z_{1} \in U$. The vector field $h(z, t)$ associated with the Loewner chain $F_{\alpha, \beta}(z, t)$ has the following form (see [8]):

$$
h(z, t)=\left(z_{1} p\left(z_{1}, t\right), z_{2}\left(1-\alpha-\beta+(\alpha+\beta) p\left(z_{1}, t\right)+\beta z_{1} p^{\prime}\left(z_{1}, t\right)\right)\right)
$$

for $z=\left(z_{1}, z_{2}\right) \in \mathbf{B}^{2}$ and $t \geq 0$. This expression was obtained from the Loewner differential equation

$$
\frac{\partial F_{\alpha, \beta}}{\partial t}(z, t)=D F_{\alpha, \beta}(z, t) h(z, t) \quad \text { a.e. } t \geq 0, \forall z \in \mathbf{B}^{2} .
$$

We shall prove that $h(\cdot, t) \in \mathcal{M}_{g}$ for a.e. $t \geq 0$. Therefore, it suffices to show that the following condition holds:

$$
\frac{1}{\|z\|^{2}}\langle h(z, t), z\rangle \in g(U) \quad \text { a.e. } t \geq 0, z \in \mathbf{B}^{2} \backslash\{0\} .
$$

Next, we will consider the following cases:

Case 1. If $B=-1$ then the function $g$ becomes $g(\zeta)=(1+A \zeta) /(1-\zeta), \zeta \in U$. In this case, the function $g$ maps the unit disk onto the half-plane $\{\zeta \in \mathbb{C}: \operatorname{Re} \zeta>(1-A) / 2\}$.

The relation $(2.2)$ is equivalent to

$$
\frac{1}{\|z\|^{2}} \operatorname{Re}\langle h(z, t), z\rangle>\frac{1-A}{2} \text { a.e. } t \geq 0, z \in \mathbf{B}^{2} \backslash\{0\} .
$$

Also, in this case, the relation $p\left(z_{1}, t\right) \in g(U)$ is equivalent to $\operatorname{Re} p\left(z_{1}, t\right)>(1-A) / 2$ for a.e. $t \geq 0, z_{1} \in U$.

Without loss of generality, we may assume that $h(\cdot, t)$ is holomorphic on $\overline{\mathbf{B}}^{2}$. Otherwise, let $\rho \in(0,1)$. Also, let $h_{\rho}(z, t)=\frac{1}{\rho} h(\rho z, t)$ for all $z \in \overline{\mathbf{B}}^{2}, t \geq 0$. Then the mapping $h_{\rho}(\cdot, t)$ is well defined and holomorphic on $\overline{\mathbf{B}}^{2}, t \geq 0$. Next, if $w \in \partial \mathbf{B}^{2}$ is fixed and $t \geq 0$, then the function $q_{\rho}(\cdot, t): \bar{U} \rightarrow \mathbb{C}$ given by $q_{\rho}(\zeta, t)=\frac{1}{\zeta}\left\langle h_{\rho}(\zeta w, t), w\right\rangle$ for $\zeta \in U \backslash\{0\}$, and $q_{\rho}(0)=1$, is holomorphic on $U$ and continuous on $\bar{U}$. Thus $\operatorname{Re} q_{\rho}(\cdot, t)$ is harmonic on $U$ and continuous on $\bar{U}$.

In view of the minimum principle for harmonic functions, it suffices to prove that $\operatorname{Re} q_{\rho}(\zeta, t) \geq(1-A) / 2$ for $|\zeta|=1$. Then $\operatorname{Re} q_{\rho}(\zeta, t)>(1-A) / 2$ for $|\zeta|<1$, by the fact that $\operatorname{Re} q_{\rho}(0, t)=1>(1-A) / 2$ and since $\operatorname{Re} q_{\rho}(\cdot, t)$ is harmonic on $U$. 
Next, if $z \in \mathbf{B}^{2} \backslash\{0\}$ and $w=z /\|z\|$, then $w \in \partial \mathbf{B}^{2}$, and if $\zeta=\|z\|$, then $\zeta \in U$, and $(1-A) / 2<\operatorname{Re} q_{\rho}(\|z\|, t)=\frac{1}{\|z\|^{2}} \operatorname{Re}\left\langle h_{\rho}(z, t), z\right\rangle$. Then letting $\rho \nearrow 1$, we deduce that $\operatorname{Re}\left\langle h(z, t), z /\|z\|^{2}\right\rangle>(1-A) / 2$, by the same argument as above, based on the minimum principle for harmonic functions.

Consequently, in view of the above arguments, we have to prove that:

$$
\operatorname{Re}\langle h(z, t), z\rangle \geq \frac{1-A}{2} \quad \text { a.e. } t \geq 0, \forall z=\left(z_{1}, z_{2}\right) \in \partial \mathbf{B}^{2} .
$$

Indeed, if we fix $z=\left(z_{1}, z_{2}\right) \in \partial \mathbf{B}^{2}$ and using elementary computation, we obtain the following relation:

$$
\begin{aligned}
\operatorname{Re}\langle h(z, t), z\rangle-\frac{1-A}{2}= & {\left[\left|z_{1}\right|^{2}+\left(1-\left|z_{1}\right|^{2}\right)(\alpha+\beta)\right] \operatorname{Re} p\left(z_{1}, t\right) } \\
& +\left(1-\left|z_{1}\right|^{2}\right) \beta \operatorname{Re}\left(z_{1} p^{\prime}\left(z_{1}, t\right)\right)+\left(1-\left|z_{1}\right|^{2}\right)(1-\alpha-\beta)-\frac{1-A}{2} .
\end{aligned}
$$

It can be seen that for $\left|z_{1}\right|=1$ (which implies $z_{2}=0$ ), the above expression is non-negative. Therefore, further we consider only the case $z_{2} \neq 0$, thus $\left|z_{1}\right|<1$.

Since $\operatorname{Re}\left(z_{1} p^{\prime}\left(z_{1}, t\right)\right) \geq-\left|z_{1}\right|\left|p^{\prime}\left(z_{1}, t\right)\right|$, this implies that

$$
\begin{aligned}
\operatorname{Re}\langle h(z, t), z\rangle-\frac{1-A}{2} \geq & {\left[\left|z_{1}\right|^{2}+\left(1-\left|z_{1}\right|^{2}\right)(\alpha+\beta)\right] \operatorname{Re} p\left(z_{1}, t\right) } \\
& -\left(1-\left|z_{1}\right|^{2}\right) \beta\left|z_{1}\right|\left|p^{\prime}\left(z_{1}, t\right)\right| \\
& +\left(1-\left|z_{1}\right|^{2}\right)(1-\alpha-\beta)-\frac{1-A}{2} .
\end{aligned}
$$

Next, we wish to give an estimate of the right-hand side member of the above inequality. First, we give an upper bound for $\left|p^{\prime}\left(z_{1}, t\right)\right|$.

It can be easily seen that

$$
\frac{p(\cdot, t)-(1-A) / 2}{1-(1-A) / 2} \in \mathcal{P} \quad \text { for } t \geq 0
$$

It is known that for a function $q \in \mathcal{P}$, we have (see 24 )

$$
\left|q^{\prime}(z)\right| \leq \frac{2 \operatorname{Re} q(z)}{1-|z|^{2}}, \quad|z|<1
$$

Therefore, from (2.5) and from the above inequality, we obtain

$$
\left|p^{\prime}\left(z_{1}, t\right)\right| \leq \frac{2\left(\operatorname{Re} p\left(z_{1}, t\right)-(1-A) / 2\right)}{1-\left|z_{1}\right|^{2}}, \quad\left|z_{1}\right|<1, t \geq 0 .
$$

Using the relation (2.6) and elementary computations on the right-hand side of (2.4), we 
obtain that

$$
\begin{aligned}
\operatorname{Re}\langle h(z, t), z\rangle-\frac{1-A}{2} \geq & {\left[\left|z_{1}\right|^{2}+\left(1-\left|z_{1}\right|^{2}\right)(\alpha+\beta)\right]\left(\operatorname{Re} p\left(z_{1}, t\right)-\frac{1-A}{2}\right) } \\
& -2 \beta\left|z_{1}\right|\left(\operatorname{Re} p\left(z_{1}, t\right)-\frac{1-A}{2}\right)+\left(1-\left|z_{1}\right|^{2}\right)(1-\alpha-\beta) \\
& +\left[\left|z_{1}\right|^{2}+\left(1-\left|z_{1}\right|^{2}\right)(\alpha+\beta)\right] \frac{1-A}{2}-\frac{1-A}{2} \\
= & {\left[\left|z_{1}\right|^{2}+\left(1-\left|z_{1}\right|^{2}\right)(\alpha+\beta)-2 \beta\left|z_{1}\right|\right]\left(\operatorname{Re} p\left(z_{1}, t\right)-\frac{1-A}{2}\right) } \\
& +\left(1-\left|z_{1}\right|^{2}\right)(1-\alpha-\beta)\left(1-\frac{1-A}{2}\right) .
\end{aligned}
$$

Next, we shall prove that the following inequality holds:

$$
\begin{aligned}
& {\left[\left|z_{1}\right|^{2}+\left(1-\left|z_{1}\right|^{2}\right)(\alpha+\beta)-2 \beta\left|z_{1}\right|\right]\left(\operatorname{Re} p\left(z_{1}, t\right)-\frac{1-A}{2}\right) } \\
+ & \left(1-\left|z_{1}\right|^{2}\right)(1-\alpha-\beta)\left(1-\frac{1-A}{2}\right) \geq 0 .
\end{aligned}
$$

Indeed, in view of the following relations:

$$
\left(1-\left|z_{1}\right|^{2}\right)(1-\alpha-\beta)\left(1-\frac{1-A}{2}\right)=\left(1-\left|z_{1}\right|^{2}\right)(1-\alpha-\beta) \frac{1+A}{2} \geq 0
$$

and

$$
\left|z_{1}\right|^{2}+\left(1-\left|z_{1}\right|^{2}\right)(\alpha+\beta)-2 \beta\left|z_{1}\right|=\left(1-\left|z_{1}\right|^{2}\right) \alpha+\beta\left(1-\left|z_{1}\right|\right)^{2}+\left|z_{1}\right|^{2}(1-2 \beta) \geq 0
$$

we obtain 2.7), as desired. Taking into account the above arguments, the inequality 2.3 holds.

Case 2. If $B \neq-1$ then the function $g: U \rightarrow \mathbb{C}$ given by $g(\zeta)=(1+A \zeta) /(1+B \zeta)$, $\zeta \in U$, maps the unit disk onto the disk $U\left((1-A B) /\left(1-B^{2}\right),(A-B) /\left(1-B^{2}\right)\right)$.

To simplify the calculations we make the following notations: $a \stackrel{\text { not }}{=}(1-A B) /\left(1-B^{2}\right)$ and $b \stackrel{\text { not }}{=}(A-B) /\left(1-B^{2}\right)$.

We remark that, for a.e. $t \geq 0$ and $\forall z_{1} \in U$, the condition $p\left(z_{1}, t\right) \in g(U)$ is equivalent to $\left|p\left(z_{1}, t\right)-a\right|<b$.

In this case, the relation 2.2 is equivalent to

$$
\left|\frac{1}{\|z\|^{2}}\langle h(z, t), z\rangle-a\right|<b \quad \text { a.e. } t \geq 0, \forall z \in \mathbf{B}^{2} \backslash\{0\} .
$$

Using an argument similar to that from the beginning of the first step, we may assume that $h(\cdot, t)$ is holomorphic on $\overline{\mathbf{B}}^{2}$, and show that

$$
|\langle h(z, t), z\rangle-a| \leq b \quad \text { a.e. } t \geq 0, \forall z \in \partial \mathbf{B}^{2} .
$$


Otherwise, we replace the mapping $h(\cdot, t)$ by the mapping $h_{\rho}(z, t)=\frac{1}{\rho} h(\rho z, t)$, for $z \in \overline{\mathbf{B}}^{2}$, a.e. $t \geq 0$, where $\rho \in(0,1)$. Using a similar argument as in the Case I, we have to prove that $\left|\left\langle h_{\rho}(z, t), z\right\rangle-a\right| \leq b$ for a.e. $t \geq 0$ and $\forall z \in \partial \mathbf{B}^{2}$. Then letting $\rho \rightarrow 1$, we obtain the conclusion.

Therefore, it suffices to prove that

$$
|\langle h(z, t), z\rangle-a| \leq b \quad \text { a.e. } t \geq 0, \forall z=\left(z_{1}, z_{2}\right) \in \partial \mathbf{B}^{2} .
$$

In the case $z_{2}=0$, it is easily seen that the relation 2.9 holds, since $\left|p\left(z_{1}, t\right)-a\right| \leq b$, $\left|z_{1}\right|=1$ and a.e. $t \geq 0$.

Next, we consider $z_{2} \neq 0$ which leads to $\left|z_{1}\right|<1$. Taking into account the following relations:

$$
\begin{aligned}
|\langle h(z, t), z\rangle-a|=\mid & \left.\left.|| z_{1}\right|^{2}+\left(1-\left|z_{1}\right|^{2}\right)(\alpha+\beta)\right] p\left(z_{1}, t\right)+\left(1-\left|z_{1}\right|^{2}\right) \beta z_{1} p^{\prime}\left(z_{1}, t\right) \\
& +\left(1-\left|z_{1}\right|^{2}\right)(1-\alpha-\beta)-a \mid \\
= & \mid\left[\left|z_{1}\right|^{2}+\left(1-\left|z_{1}\right|^{2}\right)(\alpha+\beta)\right]\left(p\left(z_{1}, t\right)-a\right)+\left(1-\left|z_{1}\right|^{2}\right) \beta z_{1} p^{\prime}\left(z_{1}, t\right) \\
& \quad+\left(1-\left|z_{1}\right|^{2}\right)(1-\alpha-\beta)+\left[\left|z_{1}\right|^{2}+\left(1-\left|z_{1}\right|^{2}\right)(\alpha+\beta)\right] \cdot a-a \mid \\
=\mid & \mid\left[\left|z_{1}\right|^{2}+\left(1-\left|z_{1}\right|^{2}\right)(\alpha+\beta)\right]\left(p\left(z_{1}, t\right)-a\right)+\left(1-\left|z_{1}\right|^{2}\right) \beta z_{1} p^{\prime}\left(z_{1}, t\right) \\
& \quad+\left(1-\left|z_{1}\right|^{2}\right)(1-\alpha-\beta)(1-a) \mid,
\end{aligned}
$$

we have the following estimate:

$$
\begin{aligned}
|\langle h(z, t), z\rangle-a| \leq & {\left[\left|z_{1}\right|^{2}+\left(1-\left|z_{1}\right|^{2}\right)(\alpha+\beta)\right]\left|p\left(z_{1}, t\right)-a\right| } \\
& +\left(1-\left|z_{1}\right|^{2}\right) \beta\left|z_{1}\right|\left|p^{\prime}\left(z_{1}, t\right)\right|+\left(1-\left|z_{1}\right|^{2}\right)(1-\alpha-\beta)|1-a| .
\end{aligned}
$$

Fix $t \geq 0$ and let the function $w(\cdot, t): U \rightarrow \mathbb{C}$ be given by $w\left(z_{1}, t\right)=\left(p\left(z_{1}, t\right)-a\right) / b$, $z_{1} \in U$. Then $w(\cdot, t) \in H(U), w(0, t)=0$ and $\left|w\left(z_{1}, t\right)\right|<1,\left|z_{1}\right|<1$. Hence the function $w(\cdot, t)$ satisfies the condition of Schwarz-Pick lemma and therefore

$$
\left|p^{\prime}\left(z_{1}, t\right)\right| \leq b \cdot \frac{1-\left|p\left(z_{1}, t\right)-a\right|^{2} / b^{2}}{1-\left|z_{1}\right|^{2}}, \quad t \geq 0 .
$$

Using the above estimate, we obtain

$$
\begin{aligned}
|\langle h(z, t), z\rangle-a| \leq & {\left[\left|z_{1}\right|^{2}+\left(1-\left|z_{1}\right|^{2}\right)(\alpha+\beta)\right]\left|p\left(z_{1}, t\right)-a\right| } \\
& +b \beta\left|z_{1}\right|\left(1-\frac{\left|p\left(z_{1}, t\right)-a\right|^{2}}{b^{2}}\right)+\left(1-\left|z_{1}\right|^{2}\right)(1-\alpha-\beta)|1-a| .
\end{aligned}
$$

Next we will show that

$$
\begin{aligned}
& {\left[\left|z_{1}\right|^{2}+\left(1-\left|z_{1}\right|^{2}\right)(\alpha+\beta)\right]\left|p\left(z_{1}, t\right)-a\right|+b \beta\left|z_{1}\right|\left(1-\frac{\left|p\left(z_{1}, t\right)-a\right|^{2}}{b^{2}}\right) } \\
+ & \left(1-\left|z_{1}\right|^{2}\right)(1-\alpha-\beta)|1-a|-b \leq 0 .
\end{aligned}
$$


It is clear that the above inequality is equivalent to the following:

$$
\begin{aligned}
& {\left[\left|z_{1}\right|^{2}+\left(1-\left|z_{1}\right|^{2}\right)(\alpha+\beta)\right] \frac{\left|p\left(z_{1}, t\right)-a\right|}{b}+\beta\left|z_{1}\right|\left(1-\frac{\left|p\left(z_{1}, t\right)-a\right|^{2}}{b^{2}}\right) } \\
+ & \left(1-\left|z_{1}\right|^{2}\right)(1-\alpha-\beta) \frac{|1-a|}{b}-1 \leq 0 .
\end{aligned}
$$

We make the following notation $x:=\left|p\left(z_{1}, t\right)-a\right| / b$. Then $x \in[0,1]$. Also, let $E(x)$ be the following quantity

$$
\begin{aligned}
E(x)= & -\beta\left|z_{1}\right| x^{2}+\left[\left|z_{1}\right|^{2}+\left(1-\left|z_{1}\right|^{2}\right)(\alpha+\beta)\right] x \\
& +\left(1-\left|z_{1}\right|^{2}\right)(1-\alpha-\beta) \frac{|1-a|}{b}+\beta\left|z_{1}\right|-1 .
\end{aligned}
$$

We aim to show that $E(x) \leq 0$ for $x \in[0,1]$.

Indeed, it can be easily seen that $E(x)$ is an increasing function on the variable $x$. Therefore $E(x) \leq E(1), x \in[0,1]$. Further, we need to evaluate the sign of $E(1)$.

$$
\begin{aligned}
E(1) & =-\beta\left|z_{1}\right|+\left|z_{1}\right|^{2}+\left(1-\left|z_{1}\right|^{2}\right)(\alpha+\beta)+\left(1-\left|z_{1}\right|^{2}\right)(1-\alpha-\beta) \frac{|1-a|}{b}+\beta\left|z_{1}\right|-1 \\
& =\left(1-\left|z_{1}\right|^{2}\right)(1-\alpha-\beta)\left(\frac{|1-a|}{b}-1\right) .
\end{aligned}
$$

Replacing the constant $a$ by $(1-A B) /\left(1-B^{2}\right)$, and the constant $b$ by $(A-B) /\left(1-B^{2}\right)$, we deduce that

$$
E(1)=\left(1-\left|z_{1}\right|^{2}\right)(1-\alpha-\beta)(|B|-1) \leq 0,
$$

where we have used the fact that $\left|z_{1}\right|<1, \alpha+\beta \leq 1$ and $B \in(-1,1)$. Therefore, combining the above relations, we obtain that $E(x) \leq 0$ for $x \in[0,1]$. Thus, we conclude that the condition 2.8 is fulfilled.

Finally, since $\left\{e^{-t} f(\cdot, t)\right\}_{t \geq 0}$ is a normal family on $U$, it suffices to use arguments similar to those used in the proof of 8 . Theorem 2.1], to deduce that $\left\{e^{-t} F_{\alpha, \beta}(\cdot, t)\right\}_{t \geq 0}$ is a normal family on unit ball $\mathbf{B}^{n}$.

In view of the above arguments, we have proved that $F_{\alpha, \beta}(z, t)$ is a $g$-Loewner chain. Hence $\Phi_{n, \alpha, \beta}(f)=F_{\alpha, \beta}(\cdot, 0)$ has $g$-parametric representation on $\mathbf{B}^{n}$. This completes the proof.

As a consequence of Theorem 2.1. we shall prove that the operator $\Phi_{n, \alpha, \beta}$ preserves the notion of $g$-starlikeness on $\mathbf{B}^{n}$, where $g(\zeta)=(1+A \zeta) /(1+B \zeta), \zeta \in U$, and $-1 \leq$ $B<A \leq 1$. Particular cases of this result were obtained in 8 (for $A=1$ and $B=-1$ ) and [1] (for $A=1$ and $B=2 \gamma-1$, where $\gamma \in(0,1)$ ).

Corollary 2.2. Let $g: U \rightarrow \mathbb{C}$ be given by $g(\zeta)=(1+A \zeta) /(1+B \zeta), \zeta \in U$, where $-1 \leq B<A \leq 1$. Also, let $f \in S_{g}^{*}$. Then $F=\Phi_{n, \alpha, \beta}(f) \in S_{g}^{*}\left(\mathbf{B}^{n}\right)$ for $\alpha \in[0,1]$, $\beta \in[0,1 / 2]$ and $\alpha+\beta \leq 1$. 
Proof. Since $f \in S_{g}^{*}$, it follows that $f\left(z_{1}, t\right)=e^{t} f\left(z_{1}\right)$ is a $g$-Loewner chain (see 7$]$ ). The mapping $F_{\alpha, \beta}(z, t)$ given by 2.1 is a $g$-Loewner chain, accordingly to Theorem 2.1. But it is easy to deduce that $F_{\alpha, \beta}(z, t)=e^{t} F(z)$ for $z \in \mathbf{B}^{n}$ and $t \geq 0$. Hence $F \in S_{g}^{*}\left(\mathbf{B}^{n}\right)$. This completes the proof.

By choosing suitable values for $A, B$, we obtain the following particular cases of Corollary 2.2. These particular cases have been approached in 30 .

Corollary 2.3. (cf. [30]) Let $a, b \in \mathbb{R}$ be such that $|1-a|<b \leq a$ and let $f \in S^{*}(a, b)$. Then $F=\Phi_{n, \alpha, \beta}(f) \in S^{*}\left(a, b, \mathbf{B}^{n}\right)$ for $\alpha \in[0,1], \beta \in[0,1 / 2]$ and $\alpha+\beta \leq 1$.

Proof. Indeed, if $A=(a-1) / b, B=\left(a^{2}-b^{2}-a\right) / b$ then $S_{g}^{*}\left(\mathbf{B}^{n}\right)=S^{*}\left(a, b, \mathbf{B}^{n}\right)$, where $g$ is given by 1.1). From Corollary 2.2, we deduce that $\Phi_{n, \alpha, \beta}(f) \in S^{*}\left(a, b, \mathbf{B}^{n}\right)$ whenever $f \in S^{*}(a, b)$.

Corollary 2.4. (cf. [30]) Let $a, b \in \mathbb{R}$ be such that $|1-a|<b \leq a$ and let $f \in \mathcal{A} S^{*}(a, b)$. Then $F=\Phi_{n, \alpha, \beta}(f) \in \mathcal{A} S^{*}\left(a, b, \mathbf{B}^{n}\right)$ for $\alpha \in[0,1], \beta \in[0,1 / 2]$ and $\alpha+\beta \leq 1$.

Proof. Indeed, if the function $g$ is given by (1.1) and $A, B$ are $\left(a-a^{2}+b^{2}\right) / b$, respectively $(1-a) / b$, then $S_{g}^{*}\left(\mathbf{B}^{n}\right)=\mathcal{A} S^{*}\left(a, b, \mathbf{B}^{n}\right)$. In view of Corollary 2.2 , we have that $\Phi_{n, \alpha, \beta}(f) \in$ $\mathcal{A} S^{*}\left(a, b, \mathbf{B}^{n}\right)$ whenever $f \in \mathcal{A} S^{*}(a, b)$.

On the other hand, we mention the following well known results, which can be obtained from Corollary 2.2 for suitable values of $A$ and $B$.

Remark 2.5. (i) In the case that $A=1$ and $B=2 \gamma-1$, where $\gamma \in(0,1)$, it can be seen that $S_{g}^{*}\left(\mathbf{B}^{n}\right)$ reduces to the set $S_{\gamma}^{*}\left(\mathbf{B}^{n}\right)$ of starlike mappings of order $\gamma$ on $\mathbf{B}^{n}$. Hence we deduce that $\Phi_{n, \alpha, \beta}\left(S_{\gamma}^{*}\right) \subset S_{\gamma}^{*}\left(\mathbf{B}^{n}\right)$. This result was obtained by Hamada, Kohr and Kohr [15], in the case of $\alpha=0, \beta=\gamma=1 / 2$, and by Liu [19], in the case $\gamma \in(0,1)$ and $\alpha \in[0,1], \beta \in[0,1 / 2], \alpha+\beta \leq 1$. T. Chirilă proved this result by using $g$-Loewner chains (see [1]).

(ii) Also, if $A=1$ and $B=-1$ then $S_{g}^{*}\left(\mathbf{B}^{n}\right)$ reduces to the set $S^{*}\left(\mathbf{B}^{n}\right)$ of normalized starlike mappings on $\mathbf{B}^{n}$. In view of Corollary 2.2 , it can be seen that the operator $\Phi_{n, \alpha, \beta}$ has the property that $\Phi_{n, \alpha, \beta}\left(S^{*}\right) \subset S^{*}\left(\mathbf{B}^{n}\right)$. This result was obtained in [8].

The following result can be proved by arguments similar to those used in the proof of Corollary 2.2. We omit the proof of Corollary 2.6 .

Corollary 2.6. Let $g: U \rightarrow \mathbb{C}$ be given by $g(\zeta)=(1+A \zeta) /(1+B \zeta), \zeta \in U$, where $-1 \leq B<A \leq 1$. Also, let $f$ be a g-spirallike function of type $\delta$ on the unit disk $U$, where $\delta \in(-\pi / 2, \pi / 2)$. Then $F=\Phi_{n, \alpha, \beta}(f)$ is a g-spirallike mapping of type $\delta$ on $\mathbf{B}^{n}$, with $\alpha \in[0,1], \beta \in[0,1 / 2]$ and $\alpha+\beta \leq 1$. 
Remark 2.7. If $A=1$ and $B=2 \gamma-1$, where $\gamma \in(0,1)$, then from Corollary 2.6 we deduce that the operator $\Phi_{n, \alpha, \beta}$ preserves the notion of spirallikenes of type $\delta$ and order $\gamma$ with $\delta \in(-\pi / 2, \pi / 2)$. This result was obtained in 20] (see also [1, 19, 32]).

\section{Radius problems}

In this section we are concerned with certain radius problems which involve the operator $\Phi_{n, \alpha, \beta}$ and the notion of Janowski starlikeness. Other radius problems related to the subclasses of $S\left(\mathbf{B}^{n}\right)$ generated by the Roper-Suffridge extension operator and other extension operators were obtained in [1, 11].

The proof for the following result is immediate and we omit it. For $r \in(0,1]$, let us consider the following set of biholomorphic mappings on $\mathbf{B}_{r}^{n}=\left\{z \in \mathbb{C}^{n}:\|z\|<r\right\}$ :

$$
\begin{aligned}
S^{*}\left(a, b, \mathbf{B}_{r}^{n}\right)= & \left\{f \text { a normalized locally biholomorphic mapping on } \mathbf{B}_{r}^{n}:\right. \\
& \left.\left|\frac{\|z\|^{2}}{\left\langle[D f(z)]^{-1} f(z), z\right\rangle}-a\right|<b, z \in \mathbf{B}_{r}^{n} \backslash\{0\}\right\},
\end{aligned}
$$

where $|1-a|<b \leq a$. In the case $n=1$, the set $S^{*}\left(a, b, \mathbf{B}_{r}^{n}\right)$ is denoted $S^{*}\left(a, b, U_{r}\right)$.

In the following remark (see also $[8$, Remark 5.1]), we assume that $\alpha, \beta \in[0,1]$ with $\beta \leq 1 / 2$ and $\alpha+\beta \leq 1$. Also, let $a, b \in \mathbb{R}$ be such that $|1-a|<b \leq a$.

Remark 3.1. (i) If $\Phi_{n, \alpha, \beta}(f) \in S^{*}\left(a, b, B_{r}^{n}\right)$ then $f \in S^{*}\left(a, b, U_{r}\right)$ for all $r \in(0,1)$.

(ii) If $f \in S^{*}\left(a, b, U_{r}\right)$ then $\Phi_{n, \alpha, \beta}(f) \in S^{*}\left(a, b, \mathbf{B}_{r}^{n}\right)$ for all $r \in(0,1)$. This result is due to the following equality (see [8])

$$
\Phi_{n, \alpha, \beta}\left(f_{r}\right)(z)=\frac{1}{r} \Phi_{n, \alpha, \beta}(f)(r z), \quad z \in \mathbf{B}^{n},
$$

where $f_{r}(\zeta)=\frac{1}{r} f(r \zeta), \zeta \in U$.

We will consider $a, b \in \mathbb{R}$ such that $|1-a|<b \leq a$. Further, we obtain the $S^{*}(a, b)$ radius of the class $S$ (respectively $S^{*}$ ). For suitable values of the parameters $a$ and $b$ depending on $A$ and $B$ (see [27]), where $-1 \leq B<A \leq 1$, we can obtain the $S^{*}[A, B]$ radius of the class $S$ (respectively $S^{*}$ ). The $S^{*}[A, B]$ radius was obtained in [22] on a wider class, namely the class of normalized analytical functions on the unit disk $U$ with fixed second coefficient.

First, we recall the definition of the $S^{*}(a, b)$ radius of the class $S$ (respectively $S^{*}$ ).

Definition 3.2. (cf. 27]) The $S^{*}(a, b)$ radius of $S$ (respectively $S^{*}$ ), denoted by $\rho^{*}(a, b)$ (respectively $\rho_{*}(a, b)$ ), is the radius of the largest disk $|z|<\rho^{*}(a, b)$ (respectively $\rho_{*}(a, b)$ ) in which the condition

$$
\left|\frac{z f^{\prime}(z)}{f(z)}-a\right|<b
$$


holds for all $f \in S$ (respectively $S^{*}$ ).

In order to prove the following result, we use the radius of starlikeness of the class $S$, i.e., the radius of the largest disk centered at the origin in which every function from $S$ is starlike. We denote the radius of starlikeness of the class $S$ by $r^{*}(S)$. It is well known that $r^{*}(S)=\tanh (\pi / 4) \approx 0.65579 \ldots$ (see e.g., [24, Corollary 6.3]).

Theorem 3.3. Let $a, b \in \mathbb{R}$ be such that $|1-a|<b \leq a$. Then the $S^{*}(a, b)$ radius of $S$ is given by

$$
\rho^{*}(a, b)=\min \left\{\frac{1-a+b}{1+a-b}, \frac{-1+a+b}{1+a+b}, \tanh \left(\frac{\pi}{4}\right)\right\} .
$$

Proof. We will use arguments similar to those in [27, Theorem 4]. We know that $S^{*}(a, b) \subset$ $S^{*}$, thus $\rho^{*}(a, b) \leq r^{*}(S)$. Further, let $f \in S$. From the definition of $r^{*}(S)$, we have that $f \in S^{*}\left(U_{\tanh (\pi / 4)}\right)$, where $U_{\tanh (\pi / 4)}=\{z \in \mathbb{C}:|z|<\tanh (\pi / 4)\}$. This is equivalent to

$$
\operatorname{Re} \frac{z f^{\prime}(z)}{f(z)}>0, \quad|z|<\tanh (\pi / 4) .
$$

Let $\rho \in(0,1)$. We say that the function $p: U \rightarrow \mathbb{C}$ belongs to $\mathcal{P}\left(U_{\rho}\right)$ if and only if $p_{\rho} \in \mathcal{P}$, where $p_{\rho}(z)=\frac{1}{\rho} p(\rho z), z \in U$.

It is easy to see that $z f^{\prime}(z) / f(z) \in \mathcal{P}\left(U_{\tanh (\pi / 4)}\right)$. It is known that a function $p$ from $\mathcal{P}$ satisfies the property $p\left(\bar{U}_{\rho}\right) \subseteq \bar{U}\left(\left(1+\rho^{2}\right) /\left(1-\rho^{2}\right), 2 \rho /\left(1-\rho^{2}\right)\right)$ for all $\rho \in(0,1)$ (see e.g., 10,24$)$.

Hence, $z f^{\prime}(z) / f(z)$ fulfills the next condition

$$
\left|\frac{z f^{\prime}(z)}{f(z)}-\frac{1+\rho^{2}}{1-\rho^{2}}\right| \leq \frac{2 \rho}{1-\rho^{2}}, \quad|z| \leq \rho, \rho \in(0, \tanh (\pi / 4)) .
$$

Let $|z|<\rho$ with $\rho \in(0, \tanh (\pi / 4)]$. The relation (3.1) holds if $\bar{U}\left(\left(1+\rho^{2}\right) /\left(1-\rho^{2}\right), 2 \rho /(1-\right.$ $\left.\left.\rho^{2}\right)\right) \subseteq \bar{U}(a, b)$. This implies that the following two conditions are simultaneously fulfilled

$$
\begin{aligned}
& a-b \leq \frac{1+\rho^{2}}{1-\rho^{2}}-\frac{2 \rho}{1-\rho^{2}}, \\
& a+b \geq \frac{1+\rho^{2}}{1-\rho^{2}}+\frac{2 \rho}{1-\rho^{2}} .
\end{aligned}
$$

The above inequalities are true if $\rho \leq \min \{(1-a+b) /(1+a-b),(-1+a+b) /(1+a+b)\}$. Since $\rho \leq \tanh (\pi / 4)$, we obtain in view of the above arguments that $\rho \leq r$ where

$$
r=\min \left\{\frac{1-a+b}{1+a-b}, \frac{-1+a+b}{1+a+b}, \tanh (\pi / 4)\right\} .
$$

This leads us to the fact that every $f \in S$ is also in $S^{*}\left(a, b, U_{r}\right)$. Moreover, there exists at least one function $f \in S$ such that $f \in S^{*}\left(a, b, U_{r}\right) \backslash S^{*}\left(a, b, U_{R}\right)$ for all $R>r$. This 
can be easily seen when the minimum in the expression of $r$ is attained at $\tanh (\pi / 4)$. There exists $f_{0} \in S$ such that $f_{0} \notin S^{*}\left(U_{R}\right)$ for all $R>\tanh (\pi / 4)$. Hence, in this case, if $f_{0} \in S^{*}\left(a, b, U_{R}\right)$ then this implies $f_{0} \in S^{*}\left(U_{R}\right)$. Therefore we obtain a contradiction.

If $r \neq \tanh (\pi / 4)$ then it suffices to prove the above statement by choosing the Koebe function:

$$
k(\zeta)=\frac{\zeta}{(1-\zeta)^{2}}, \quad|\zeta|<1
$$

Suppose that $k \in S^{*}\left(a, b, U_{R}\right)$ for some $R \in(r, 1)$ and derive a contradiction. In this case, the relation 3.1$)$ is equivalent to the following:

$$
\left|\frac{1+z}{1-z}-a\right|<b
$$

for $|z|<R$. We will show that for some $z_{0}$ with $\left|z_{0}\right|=r<R$ the condition (3.3) is no longer true.

If the minimum in the expression of $r$ is attained at $\rho=(1-a+b) /(1+a-b)$ then, for $z_{0}=-\rho$, we have that

$$
\begin{aligned}
\left|\frac{1+z_{0}}{1-z_{0}}-a\right| & =\left|\frac{1-(1-a+b) /(1+a-b)}{1+(1-a+b) /(1+a-b)}-a\right| \\
& =|a-b-a|=|-b|=b .
\end{aligned}
$$

Otherwise, if the minimum is attained at $\rho=(-1+a+b) /(1+a+b)$ then for $z_{0}=\rho$, it results that

$$
\begin{aligned}
\left|\frac{1+z_{0}}{1-z_{0}}-a\right| & =\left|\frac{1+(-1+a+b) /(1+a+b)}{1-(-1+a+b) /(1+a+b)}-a\right| \\
& =|a+b-a|=|b|=b
\end{aligned}
$$

Hence, in both cases, we get a contradiction to (3.3). Therefore, there exists at least one function $f \in S^{*}\left(a, b, U_{r}\right)$ such that $f \notin S^{*}\left(a, b, U_{R}\right)$ for every $R>r$. Hence, we have proved that $r$ is the radius of the largest disk in which the condition (3.1) holds. This completes the proof.

In the case that $a=b$ in Theorem 3.3 , we obtain the following particular case:

Corollary 3.4. Let $r=\frac{1}{2} \cdot e^{\pi / 2} \approx 2.4052 \ldots$ Then we have that $\rho^{*}(a, a)=(2 a-1) /(2 a+1)$ for $1 / 2<a<r$ and $\rho^{*}(a, a)=\tanh (\pi / 4)$ for $a \geq r$.

Proof. If we make the substitution $a=b$ in Theorem 3.3 then

$$
\rho^{*}(a, a)=\min \left\{1, \frac{2 a-1}{2 a+1}, \tanh \left(\frac{\pi}{4}\right)\right\} .
$$

It can easily be seen that $(2 a-1) /(2 a+1)<1$. Also, the function $(2 a-1) /(2 a+1)$ is increasing and is equal to $\tanh (\pi / 4)$ when $a=r$, where $r=\frac{1}{2} \cdot e^{\pi / 2}$. 
This corollary shows that if $f \in S$ then $f$ is not only starlike on $U_{\tanh (\pi / 4)}$, but it is also in $S^{*}\left(a, a, U_{\tanh (\pi / 4)}\right)$, when $a \geq \frac{1}{2} \cdot e^{\pi / 2}$.

In view of the proof of Theorem 3.3 , we obtain the $S^{*}(a, b)$ radius of the class $S^{*}$.

Theorem 3.5. Let $a, b \in \mathbb{R}$ be such that $|1-a|<b \leq a$. Then the $S^{*}(a, b)$ radius of $S^{*}$ is given by

$$
\rho_{*}(a, b)=\min \left\{\frac{1-a+b}{1+a-b}, \frac{-1+a+b}{1+a+b}\right\} .
$$

Moreover, if $a=b$ then $\rho_{*}(a, a)=(2 a-1) /(2 a+1)$.

In view of Remark 1.5 and Corollary 3.4, we obtain the following particular case.

Remark 3.6. Let $\gamma \in(0,1)$.

(i) The radius of almost starlikeness of order $\gamma$ of the class $S$ is $\tanh (\pi / 4)$, when $0<$ $\gamma \leq e^{-\pi / 2}$ and $(1-\gamma) /(1+\gamma)$, when $e^{-\pi / 2}<\gamma<1$.

(ii) The radius of almost starlikeness of order $\gamma$ of the class $S^{*}$ is $(1-\gamma) /(1+\gamma)$.

Assuming $|1-a|<b \leq a$, we refer to the $S^{*}(a, b)$ radius of class $\Phi_{n, \alpha, \beta}(S)$ (respectively $\left.\Phi_{n, \alpha, \beta}\left(S^{*}\right)\right)$ as the radius $r \in(0,1]$ of the largest ball $\mathbf{B}_{r}^{n}$ such that every mapping $F \in$ $\Phi_{n, \alpha, \beta}(S)$ (respectively $F \in \Phi_{n, \alpha, \beta}\left(S^{*}\right)$ ) is a member of the family $S^{*}\left(a, b, \mathbf{B}_{r}^{n}\right)$.

Theorem 3.7. If $\alpha \in[0,1], \beta \in[0,1 / 2]$ and $\alpha+\beta \leq 1$ then the $S^{*}(a, b)$ radius of $\Phi_{n, \alpha, \beta}(S)$ is $\rho^{*}(a, b)$, where $\rho^{*}(a, b)$ is given by (3.2).

Proof. Let $f \in S$. Then $f \in S^{*}\left(a, b, U_{\rho^{*}(a, b)}\right)$. We denote $F_{\alpha, \beta}=\Phi_{n, \alpha, \beta}(f)$. In view of Corollary 2.3 and Remark 3.1(ii), we have that $F_{\alpha, \beta} \in S^{*}\left(a, b, \mathbf{B}_{\rho^{*}(a, b)}^{n}\right)$. This shows that the $S^{*}(a, b)$ radius of $\Phi_{n, \alpha, \beta}(S)$ is greater than or equal to $\rho^{*}(a, b)$. From the proof of Theorem 3.3. we know that the relation (3.1) may not hold when $|z| \geq \rho^{*}(a, b)$. From Remark 3.1(i), the mapping $F_{\alpha, \beta}$ may fail to be a Janowski starlike mapping on $\mathbf{B}_{R}^{n}$ with $R>\rho^{*}(a, b)$. Hence, we conclude that $\rho^{*}(a, b)$ is the biggest radius $r$ for which every $F_{\alpha, \beta}=\Phi_{n, \alpha, \beta}(f)$ is Janowski starlike on $\mathbf{B}_{r}^{n}$.

With arguments similar to those used in the proof of Theorem 3.7, the following results hold.

Theorem 3.8. Let $\alpha, \beta \in[0,1]$ with $\beta \leq 1 / 2$ and $\alpha+\beta \leq 1$.

(i) The $S^{*}(a, b)$ radius of $\Phi_{n, \alpha, \beta}\left(S^{*}\right)$ is $\rho_{*}(a, b)$, where $\rho_{*}(a, b)$ is given by 3.4).

(ii) Let $\gamma \in(0,1)$. The radius of almost starlikeness of order $\gamma$ of $\Phi_{n, \alpha, \beta}(S)$ is $\tanh (\pi / 4)$ for $0<\gamma \leq e^{-\pi / 2}$ and $(1-\gamma) /(1+\gamma)$ for $e^{-\pi / 2}<\gamma<1$.

Also, the radius of almost starlikeness of order $\gamma$ of $\Phi_{n, \alpha, \beta}\left(S^{*}\right)$ is $(1-\gamma) /(1+\gamma)$. 


\section{Acknowledgments}

The author thanks to the anonymous referee for his careful reading and useful suggestions which improved the paper.

\section{References}

[1] T. Chirilă, An extension operator associated with certain g-Loewner chains, Taiwanese J. Math. 17 (2013), no. 5, 1819-1837.

[2] _ Subclasses of biholomorphic mappings associated with g-Loewner chains on the unit ball in $\mathbb{C}^{n}$, Complex Var. Elliptic Equ. 59 (2014), no. 10, 1456-1474.

[3] P. Curt, A Marx-Strohhäcker theorem in several complex variables, Mathematica 39(62) (1997), no. 1, 59-70.

[4] _ Janowski starlikeness in several complex variables and complex Hilbert spaces, Taiwanese J. Math. 18 (2014), no. 4, 1171-1184.

[5] P. Duren, I. Graham, H. Hamada and G. Kohr, Solutions for the generalized Loewner differential equation in several complex variables, Math. Ann. 347 (2010), no. 2, 411435.

[6] S. X. Feng, Some Classes of Holomorphic Mappings in Several Complex Variables, University of Science and Technology of China, Doctor Thesis, 2004.

[7] I. Graham, H. Hamada and G. Kohr, Parametric representation of univalent mappings in several complex variables, Canad. J. Math. 54 (2002), no. 2, 324-351.

[8] I. Graham, H. Hamada, G. Kohr and T. J. Suffridge, Extension operators for locally univalent mappings, Michigan Math. J. 50 (2002), no. 1, 37-55.

[9] I. Graham and G. Kohr, An extension theorem and subclasses of univalent mappings in several complex variables, Complex Var. Theory Appl. 47 (2002), no. 1, 59-72.

[10] _ Geometric Function Theory in One and Higher Dimensions, Monographs and Textbooks in Pure and Applied Mathematics 255, Marcel Dekker, New York, 2003.

[11] I. Graham, G. Kohr and M. Kohr, Loewner chains and the Roper-Suffridge extension operator, J. Math. Anal. Appl. 247 (2000), no. 2, 448-465.

[12] — Loewner chains and parametric representation in several complex variables, J. Math. Anal. Appl. 281 (2003), no. 2, 425-438. 
[13] H. Hamada and T. Honda, Sharp growth theorems and coefficient bounds for starlike mappings in several complex variables, Chin. Ann. Math. Ser. B 29 (2008), no. 4, 353-368.

[14] H. Hamada and G. Kohr, Subordination chains and the growth theorem of spirallike mappings, Mathematica 42(65) (2000), no. 2, 153-161.

[15] H. Hamada, G. Kohr and M. Kohr, Parametric representation and extension operators for biholomorphic mappings on some Reinhardt domains, Complex Var. Theory Appl. 50 (2005), no. 7-11, 507-519.

[16] W. Janowski, Some extremal problems for certain families of analytic functions I, Ann. Polon. Math. 28 (1973), 297-326.

[17] G. Kohr, Certain partial differential inequalities and applications for holomorphic mappings defined on the unit ball of $\mathbb{C}^{n}$, Ann. Univ. Mariae Curie-Skłodowska Sect. A 50 (1996), 87-94.

[18] - On some sufficient conditions of almost starlikeness of order $1 / 2$ in $C^{n}$, Studia Univ. Babeş-Bolyai Math. 41 (1996), no. 3, 51-55.

[19] X. Liu, The generalized Roper-Suffridge extension operator for some biholomorphic mappings, J. Math. Anal. Appl. 324 (2006), no. 1, 604-614.

[20] X. S. Liu and T. S. Liu, The generalized Roper-Suffridge extension operator for spirallike mappings of type $\beta$ and order $\alpha$, Chinese Ann. Math. Ser. A 27 (2006), no. 6, 789-798.

[21] J. R. Muir, Jr., Extension of convex mappings of order $\alpha$ of the unit disk in $\mathbb{C}$ to convex mappings of the unit ball in $\mathbb{C}^{n}$, J. Math. Anal. Appl. 356 (2009), no. 1, $369-377$.

[22] M. M. Nargesi, R. M. Ali and V. Ravichandran, Radius constants for analytic functions with fixed second coefficient, The Scientific World Journal 2014 (2014), Art. ID 898614, 6 pp.

[23] J. A. Pfaltzgraff, Subordination chains and univalence of holomorphic mappings in $C^{n}$, Math. Ann. 210 (1974), 55-68.

[24] C. Pommerenke, Univalent Functions, Studia Mathematica/Mathematische Lehrbücher, Band XXV, Vandenhoeck \& Ruprecht, Göttingen, 1975.

[25] K. A. Roper and T. J. Suffridge, Convex mappings on the unit ball of $C^{n}$, J. Anal. Math. 65 (1995), 333-347. 
[26] H. Silverman, Subclasses of starlike functions, Rev. Roumaine Math. Pures Appl. 23 (1978), no. 7, 1093-1099.

[27] H. Silverman and E. M. Silvia, Subclasses of starlike functions subordinate to convex functions, Canad. J. Math. 37 (1985), no. 1, 48-61.

[28] T. J. Suffridge, Starlikeness, convexity and other geometric properties of holomorphic maps in higher dimensions, Complex analysis (Proc. Conf., Univ. Kentucky, Lexington, Ky., 1976), 146-159, Lecture Notes in Mathematics 599, Springer, Berlin, 1977.

[29] M. I. Voda, Loewner Theory in Several Complex Variables and Related Problems, Thesis (Ph.D.)-University of Toronto (Canada), 2011.

[30] C. Wang, Y. Cui and H. Liu, New subclasses of biholomorphic mappings and the modified Roper-Suffridge operator, Chin. Ann. Math. Ser. B 37 (2016), no. 5, 691704 .

[31] Q.-H. Xu and T.-S. Liu, Löwner chains and a subclass of biholomorphic mappings, J. Math. Anal. Appl. 334 (2007), no. 2, 1096-1105.

[32] Y.-C. Zhu and M.-S. Liu, The generalized Roper-Suffridge extension operator on Reinhardt domain $D_{p}$, Taiwanese J. Math. 14 (2010), no. 2, 359-372.

Andra Manu

Faculty of Mathematics and Computer Science, Babeş-Bolyai University, 1 M.

Kogălniceanu Str., Cluj-Napoca, Romania

E-mail address: andra.manu@math.ubbcluj.ro 